Journal of Computer Science 7 (1): 80-89, 2011

ISSN 1549-3636

(C) 2011 Science Publications

\title{
Interference Mitigation in IEEE 802.15.4-A Cluster Based Scheduling Approach
}

\author{
G.M. Tamilselvan and A. Shanmugam \\ Department of Electronics and Communication Engineering, \\ Bannari Amman Institute of Technology, \\ Sathyamangalam, Erode dt Tamilnadu, 638401, India
}

\begin{abstract}
Problem statement: In universal networking environments; two or more heterogeneous communication systems coexisting in a single place. Especially, Wireless Local Area Networks (WLANs) based on IEEE 802.11b specifications and Wireless Personal Area Networks (WPANs) based on IEEE 802.15.4 specifications need to coexist in the same Industrial, Science and Medial (ISM) band. If the WPAN communication coverage is expanded using a cluster-tree network topology, then the 802.15.4 network is more susceptible to interference from neighboring WLANs. Approach: In this study, we propose an adaptive transmission power aware cluster scheduling algorithm using multiple channels in a WPAN in the presence of WLAN interference. Results: The algorithm includes node identification, channel allocation, clustering and time scheduling. To evaluate the performance of the proposed algorithm, the performance metrics such as Bit error, Packet Error Rate (PER), Throughput, Average End-End Delay and Average Jitter is measured through Qualnet simulation. PER is calculated from bit error rate. The simulation results are compared with the conventional TDMA scheme. Conclusion/Recommendations: The measurement result shows that the proposed algorithm is effective in an IEEE 802.15.4 cluster-tree network in the presence of multiple IEEE 802.11 interferers.
\end{abstract}

Key words: Clustering, coexistence, heterogeneous, Packet Error Rate (PER), WLAN and WPAN (Zigbee)

\section{INTRODUCTION}

As a low-power and low-cost technology, IEEE 802.15.4 is establishing its place on the market as an enabler for the emerging Wireless Sensor Networks (WSNs) (Petrova et al., 2006). Like IEEE 802.11b and $802.11 \mathrm{~g}$, IEEE 802.15.4 is also used in the $2.4 \mathrm{GHz}$ ISM band. Due to supporting complimentary applications, they are very likely to be collocated within the interfering range of each other and therefore their ability to coexist needs to be evaluated. In this study we focus on the coexistence between these two major wireless standards that operate in the $2.4 \mathrm{GHz}$ ISM band. Their overlapping frequency channels are shown in Fig. 1.

IEEE 802.15.4 defines the physical layer and the MAC sub layer of the OSI Zigbee stack. It supports devices that consume minimum energy and is designed for low rate, low cost applications over a short range of 30-100 m. The IEEE 802.15.4 defines three physical layers; the $2.4 \mathrm{GHz}, 868 \mathrm{MHz}$ and $915 \mathrm{MHz}$ frequency bands. The unlicensed Industrial Scientific Medical (ISM) $2.4 \mathrm{GHz}$ band is available worldwide, while the 868 and $915 \mathrm{MHz}$ bands are available in Europe and
North America respectively. A total of 27 channels with three different data rates are defined for the IEEE 802.15.4: 16 channels with a data rate of $250 \mathrm{kbps}$ at the $2.4 \mathrm{GHz}$ band, 10 channels with a data rate of 40 kbps at the $15 \mathrm{MHz}$ band and 1 channel with a data rate of $20 \mathrm{kbps}$ at the $868 \mathrm{MHz}$ band. The relationship between the IEEE $802.11 \mathrm{~b}$ (non-overlapping sets) and the IEEE 802.15.4 channels at the $2.4 \mathrm{GHz}$ is illustrated in Fig. 1.
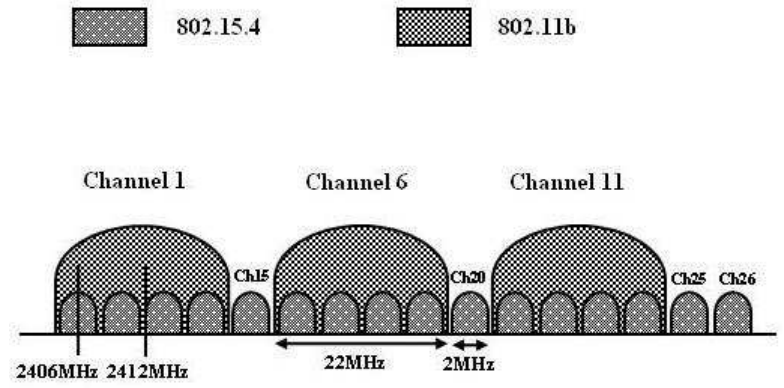

Fig. 1: 802.11 and 802.15 .4 channels in the $2.4 \mathrm{GHz}$ ISM band

Corresponding Author: G.M. Tamilselvan, Department of Electronics and Communication Engineering, Bannari Amman Institute of Technology, Sathyamangalam, Erode dt, Tamilnadu, 638401, India 
Figure 1 shows the operation frequency spectrum of both IEEE 802.11 and IEEE 802.15.4 networks in the $2.4 \mathrm{GHz}$ ISM band. The IEEE 802.11 standard has 11 channels each of which occupies $22 \mathrm{MHz}$ and up to 3 channels can be used simultaneously without mutual interference. As illustrated in the figure.1, channels 1, 6 and 11 can be used by the IEEE 802.11 devices to eliminate the mutual interference. On the other hand, the IEEE 802.15.4 standard defines 16 channels (2 $\mathrm{MHz}$ ), channels 11 through 27 , in the $2.4 \mathrm{GHz}$ ISM band all of which can be used simultaneously without mutual interference.

The IEEE 802.15.4 standard recommends using the channels that fall in the guard bands between two of the three adjacent non-overlapping IEEE 802.11 channels or above these channels to prevent interference between the IEEE 802.15.4 and the IEEE 802.11. From the Fig. 1, it is shown that 4 of the 11 channels will have the minimal interference which in most cases is enough to cover a big region unless more IEEE 802.15.4 networks are added.

There have been some studies about coexistence between the IEEE 802.11b and IEEE 802.15.4. According to (Petrova et al., 2006; Howitt and Gutierrez, 2003; Sikora and Groza, 2005) IEEE 802.15.4 has a little impact on the IEEE 802.11 performance. However, IEEE 802.11 can have a serious impact on the IEEE 802.15.4 performance if the channel allocation is not carefully taken into account (Petrova et al., 2006; Shin et al., 2005b). While the conclusion is true in general, we believe the studies so far have dealt with only limited cases of coexistence scenarios. In (Shin et al., 2005b), the Packet Error Rate (PER) of IEEE 802.15.4 under the IEEE 802.11b interference is analyzed from an assumption of blind transmissions, i.e., both IEEE 802.11b and IEEE 802.15.4 transmit packets regardless of whether the channel state is busy or not. In (Sikora and Groza, 2005), measurements are performed to quantify coexistence issues. Channel conflict probabilities between IEEE 802.15 based Wireless Personal Area Networks is modeled in (Chen et al., 2006). Packet Error Rate of IEEE 802.15.4 under IEEE 802.11b interference is analyzed in (Shin et al., 2005a). In (Yoon et al., 2006) Packet error rate of IEEE 802.11b under IEEE 802.15.4 interference is analyzed.

The author concluded that despite its low transmit power and simple modulation technique, IEEE 802.15.4 shows a robust behavior against interference of other $2.4 \mathrm{GHz}$ systems and even in the worst case conditions for frequency overlap, local distance and high traffic load for interference, some time slots remain for a successful transmission of IEEE 802.15.4. In above said related works only two WPAN nodes which are collocated with multiple WLAN nodes are considered. But today the sensor networks play a vital role in any automation; we have to consider the multiple WPAN nodes. When multiple sensor nodes are used, time slot mechanism is not helpful in WPAN network because ZigBee is a mesh networking technology.

The remainder of the study is organized as follows: The next section draws an overview of the IEEE 802.11b and IEEE 802.15.4 standard. The materials and methods section furnishes a heterogeneous wireless network with conventional TDMA scheme for packet transmission and cluster tree network with two different time and frequency scheduling schemes. The results section provides the simulation results for the three different scheduling algorithms. The reasoning for performance enhancement is presented in discussion section. In final section the conclusion is presented.

\section{Overview of IEEE 802.11b and IEEE 802.15.4:}

IEEE 802.11b: IEEE 802.11b standard defines the Medium Access Control (MAC) sub layer and the Physical (PHY) layer for wireless LANs. The standard operates at 13 overlapping channels in the $2.4 \mathrm{GHz}$ ISM band and the bandwidth of each channel is $22 \mathrm{MHz}$. IEEE 802.11b MAC employs the Carrier Sense Multiple Access with Collision Avoidance (CSMA/CA) mechanism. Before initiating a transmission, an IEEE 802.11 b node senses the channel to determine whether another node is transmitting. If the medium is sensed idle for a Distributed coordination function Inter-Frame Space (DIFS) time interval the transmission will be preceded. If the medium is busy the node defers its transmission. When the medium becomes idle for a DIFS interval, the node will generate a random back off delay uniformly chosen in an interval. This interval [0, $\mathrm{W}]$ is called Contention Window, where $\mathrm{W}$ is the size of the contention window. The initial $\mathrm{W}$ is set to CWmin. The back off timer is decreased by one as long as the medium is sensed idle for a back off time slot. The back off counter will become frozen when a transmission is detected on the medium and resumed when the channel is sensed idle again for a DIFS interval. When the back off timer reaches zero, the node transmits a DATA packet. Immediately after receiving a packet correctly, the destination node waits for a Short Inter Frame Spacing (SIFS) interval and then transmits an ACK back to the source node.

IEEE 802.15.4: The IEEE 802.15.4 MAC sub layer is based on Channel Sense Multiple Access (CSMA/CA) with two modes of operation: The unslotted-CSMA 


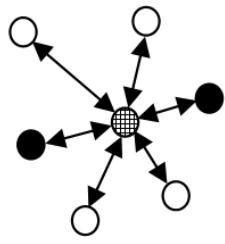

(a) Star Topology

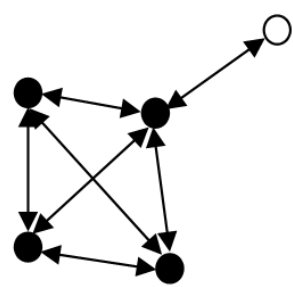

(b) Peer-Peer Topology

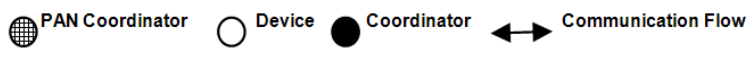

Fig. 2: IEEE 802.15.4 topologies

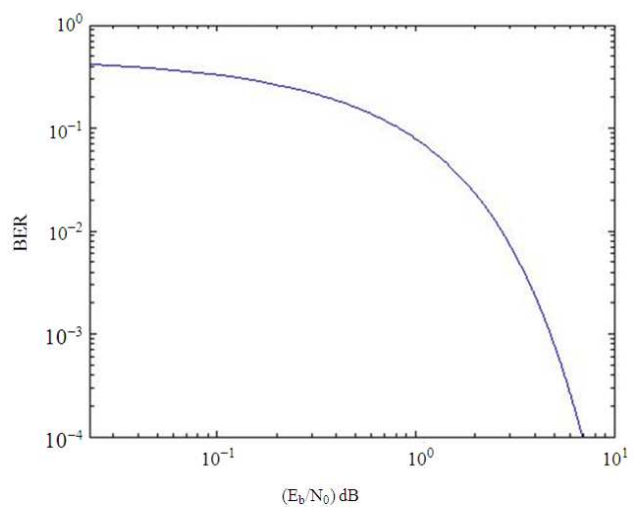

Fig. 3: Theoretic bit error rate of OQPSK

(beaconless mode) and the slotted-CSMA (beacon enabled mode). The basic responsibilities for the MAC sub layer is transmitting beacon frames, synchronization and providing a reliable transmission between Zigbee devices. Link layer acknowledgments are optional in IEEE 802.15.4 which can provide extra link level reliability. For our simulations, the unslottedCSMA is used as all sources will be continuously contending for the channel. Link layer acknowledgments are used in order to make the transmission more reliable. To minimize the energy consumption of the Zigbee nodes, the slotted CSMA/CA should be taken into consideration since it uses beacon frames that contain information about when nodes can go into sleep mode. However, this is beyond the scope of this study.

Proposed scheme: In this study, we propose power aware time slot and frequency based spectrum access analysis for the performance metrics such as bit error, PER, throughput, average End-End delay and average jitter of IEEE 802.15.4. In this proposed scheme the WPAN devices are clustered. Each cluster will have one PAN coordinator and four end devices. We consider a heterogeneous network with random topology. IEEE 802.15.4 topologies are shown in Fig. 2. Here the performance of IEEE 802.15.4 under the interference of IEEE $802.11 \mathrm{~b}$ and the interference among IEEE 802.15.4 nodes because of multiple transmissions is analyzed using Qualnet 4.5 simulation. For simulation, the unslotted CSMA/CA of the IEEE 802.15.4 model is developed using Qualnet 4.5. The random topology scenario of coexistence heterogeneous network with 20 and 20 WLAN nodes for heterogeneous wireless network with conventional TDMA scheme and two different scheduling schemes are shown in Fig. 4-6 respectively.

The PHY of the IEEE 802.15.4 at $2.4 \mathrm{GHz}$ uses Offset Quadrature Phase Shift Keying (OQPSK) modulation. Denote that the $\mathrm{E}_{\mathrm{b}} / \mathrm{N}_{0}$ is the ratio of the average energy per information bit to the noise power spectral density at the receiver input, in the case of an Additive White Gaussian Noise (AWGN) channel. Then the Bit Error Rate (BER), $\mathrm{P}_{\mathrm{B}}$, can be expressed as:

$$
\mathrm{P}_{\mathrm{B}}=\mathrm{Q}\left(\sqrt{\frac{2 \mathrm{E}_{\mathrm{b}}}{\mathrm{N}_{0}}}\right)
$$

where, $\mathrm{Q}(\mathrm{x})$ is:

$$
\mathrm{Q}(\mathrm{x})=\frac{1}{\sqrt{\mathrm{x}}} \int_{\mathrm{x}}^{\infty} \exp \left(\frac{-\mathrm{u}^{2}}{2}\right) \mathrm{du}
$$

Figure 3 shows the relationship between the bit error rate and $\mathrm{E}_{\mathrm{b}} / \mathrm{N}_{\mathrm{o}}$ simulated in MATLAB. The bit error rate decreases when $E_{b} / N_{o}$ increases. The noise power spectral density increases when collision increases. As the number of WLAN sources increases, the BER of IEEE 802.15.4 increases because contentions among multiple WLANs increase the channel usage and cause collisions, which is more powerful interference, source to.

The PER is calculated as a function of the BER, i.e., $\mathrm{P}_{\mathrm{b}}$. The probability of not having a bit error is the probability that all the bits are received correctly. Therefore the conditional probability of PER is one minus the probability of no bit errors and is computed as follows:

PER $=1-\left(1-\overline{\mathrm{P}_{\mathrm{b}}}\right)^{\mathrm{N}}$

where, $\mathrm{N}$ represents the number of bits in a packet. For the experimental setting each packet is composed of 105 bytes in the case of WPAN node and 1500 bytes in the case of WLAN node. If there is an error correction mechanism, then the PER utilizing the BER should be computed differently. However, the experimental platform does not provide an error correction mechanism and Equation 3 is the final form of the PER. 


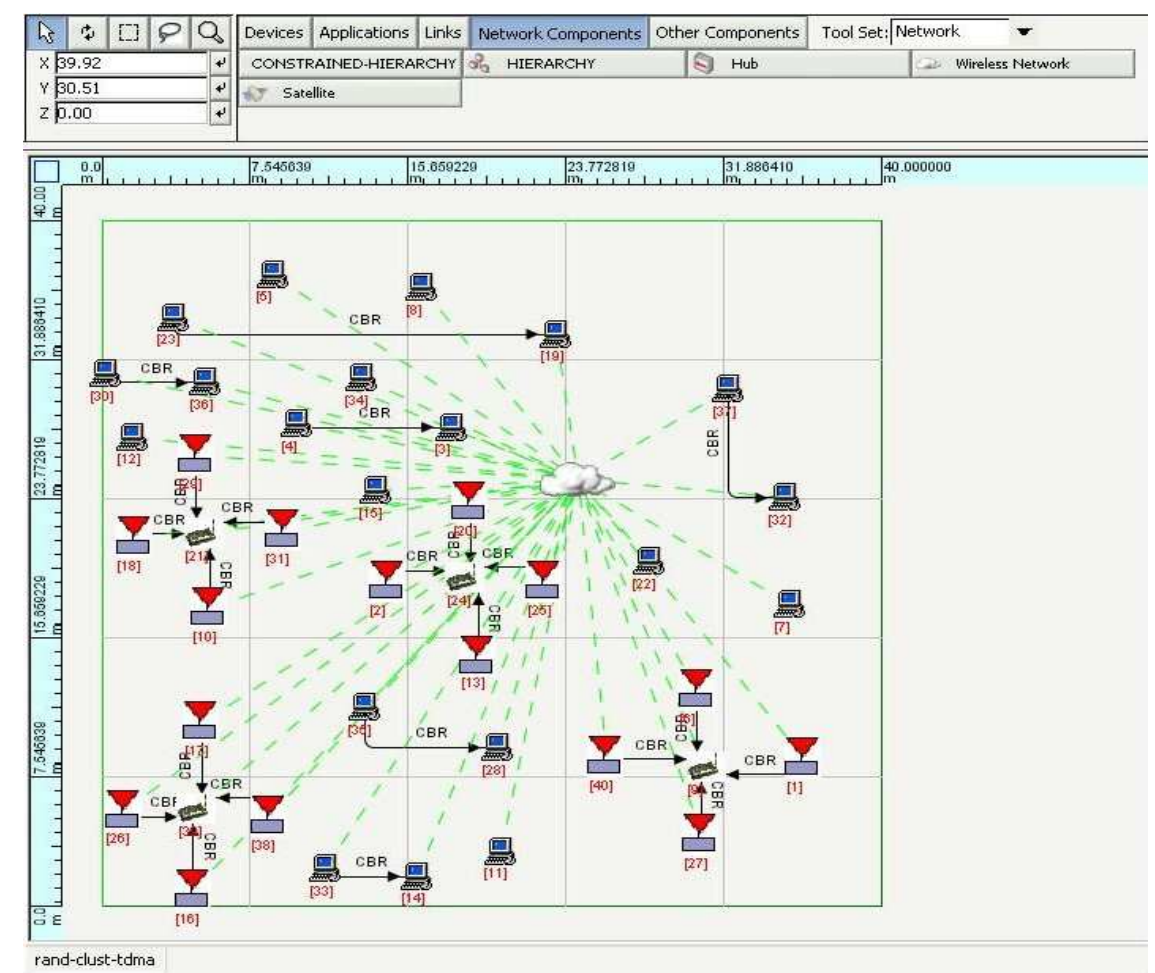

Fig. 4: Random topology scenario with conventional TDMA

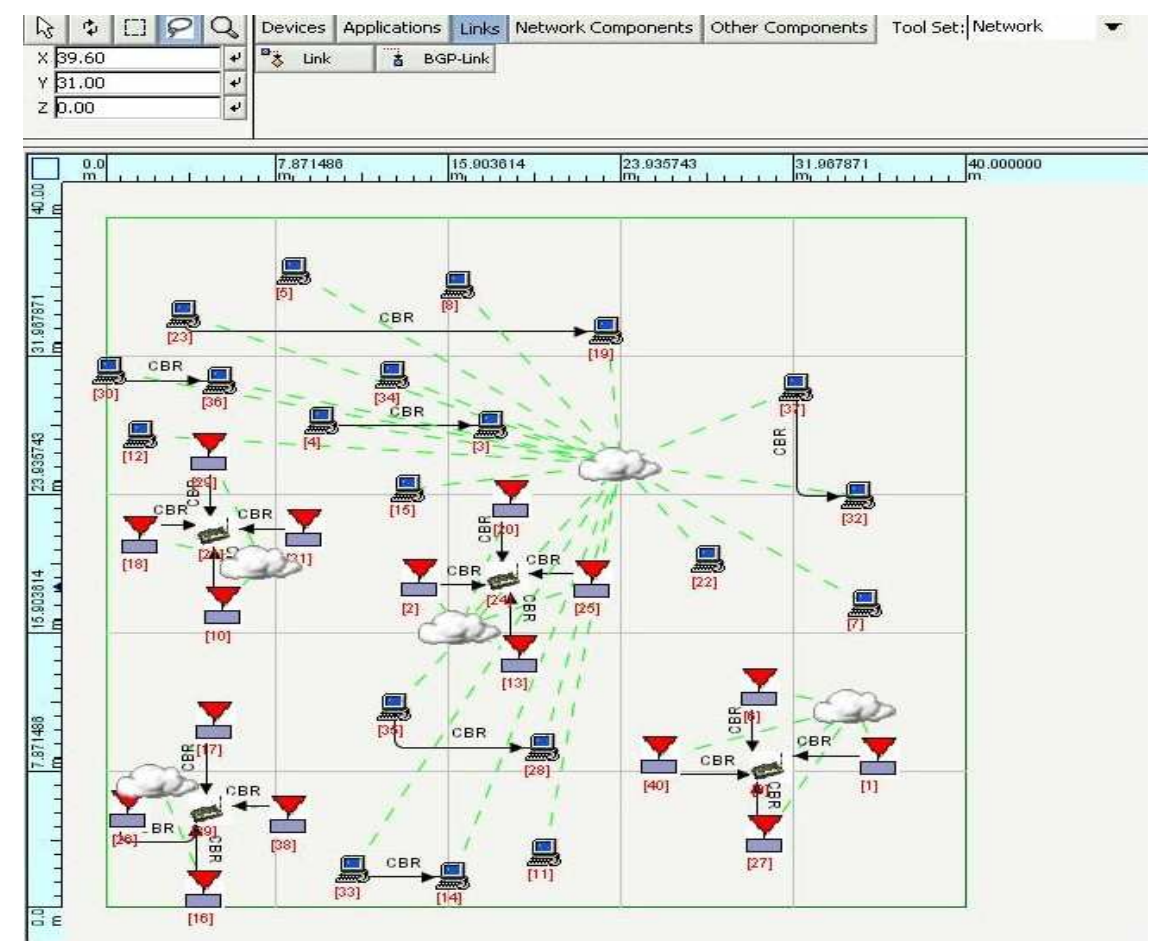

Fig. 5: Random topology scenario with Inter cluster scheduling 


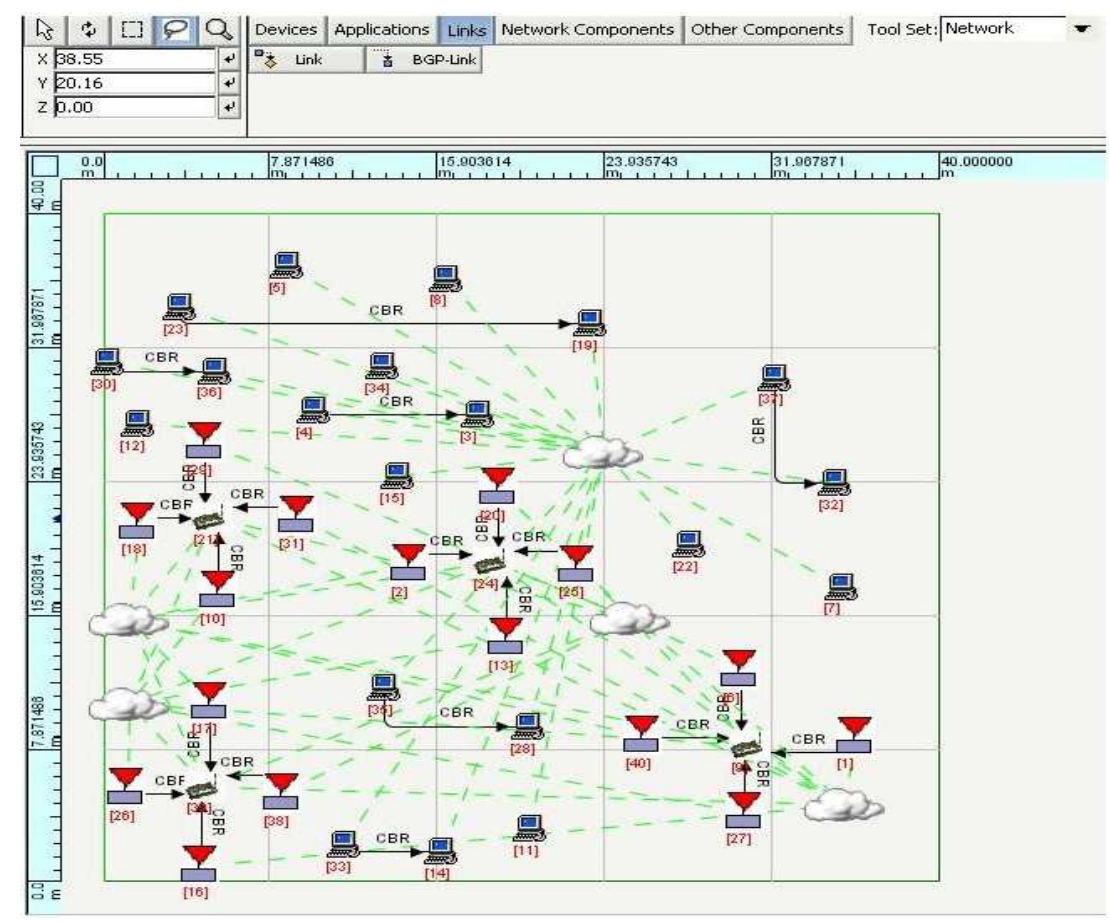

Fig. 6: Random topology scenario with Intra cluster scheduling

In this study conventional TDMA scheme and two clusters based scheduling schemes are proposed and the results are compared. In cluster based scheduling the first scheme is called intra cluster scheduling. In this scheme the nodes are separated based on their transmission power. The output power of 802.15.4 devices is typically as low as $0 \mathrm{dBm}$, whereas the output power of $802.11 \mathrm{~b}$ devices is $15 \mathrm{dBm}$ or above. Then WLAN nodes are grouped under one operating frequency and WPAN nodes are clustered with cluster size 5.Each cluster will have one PAN coordinator and four end devices. Each cluster is allotted unique channel frequency for error free transmission. After frequency scheduling, in each channel specific time slot is allotted for packet transmission.

In second scheme cluster reformation scheduling is considered. The cluster members from different clusters are grouped under one channel and specific time slots are allotted for packet transmission. The Fig. 4-6 shows the scenario for heterogeneous network with conventional TDMA scheme and two different clusters based scheduling schemes respectively.

\section{MATERIALS AND METHODS}

Scheduling algorithm: In this study the two different devices namely IEEE 802.11b and IEEE 802.15.4 is used with random topology. Three different algorithms are used and the performance of the IEEE 802.15.4 is analyzed:

- Conventional TDMA

- Intra cluster scheduling

- Reformed cluster scheduling

Conventional TDMA: The conventional time scheduling algorithm is applied for all transmissions as shown in Fig. 4. The algorithm is briefly explained as follows:

Step 1: Get the Number of Nodes

Step 2: Check for its CBR transmission

Step 3: Check for its transmission power

Step 4: If the transmission power is between $15 \mathrm{dbm}$ and $20 \mathrm{dBm}$, the node = IEEE 802.11b (WLAN) node. Assign the transmission time and sleeping time as $5 \mathrm{sec}$ and $1 \mathrm{sec}$ respectively

Step 5: If the transmission power is between $0 \mathrm{dBm}$ and $3 \mathrm{dBm}$, the node = IEEE 802.15.4 (WPAN) node. Assign the transmission time and sleeping time as 1 and $0.1 \mathrm{sec}$ respectively

Step 6: If the transmission power is not satisfying the above two cases the node is identified as invalid node and assigned sleeping time as total simulation time 
The above steps can be written as:

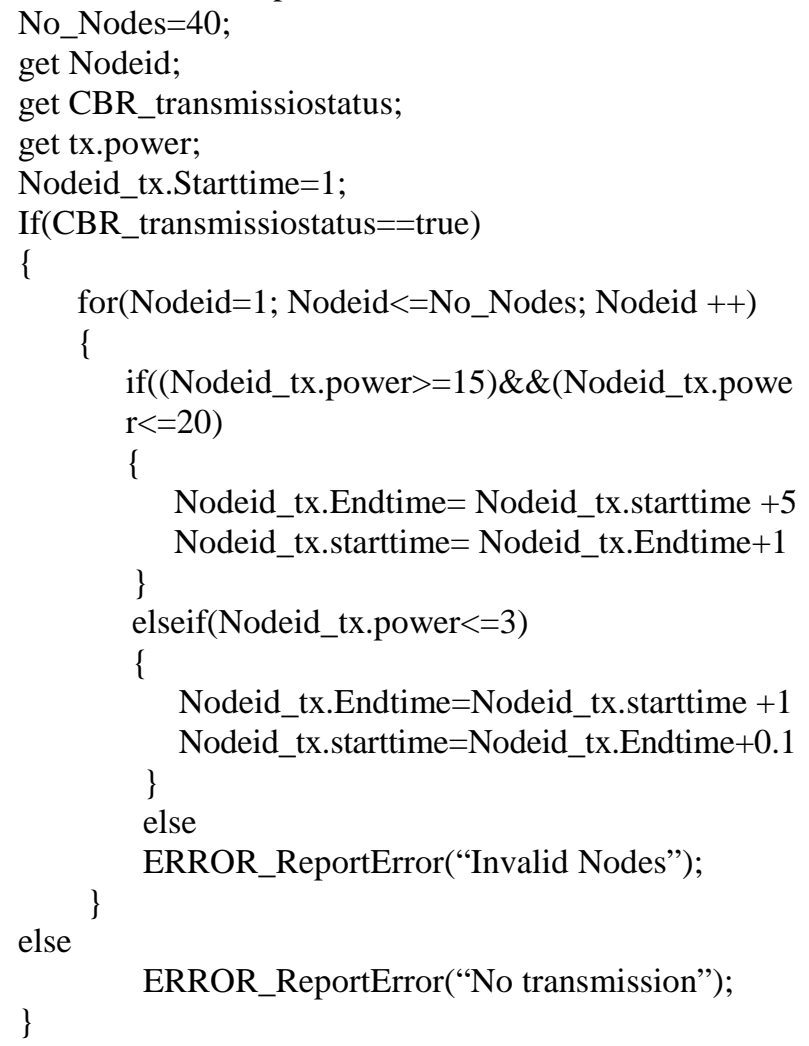

Intra cluster scheduling: In this scheduling scheme the nodes are grouped under different frequencies depend on its transmission power initially. Then low power nodes IEEE 802.15.4 are clustered with 4 cluster members and 1 cluster head. End devices are cluster members and coordinators are cluster head. Each cluster is allotted different frequencies. In each cluster the end devices transmit to the coordinator. For each transmission specific time slots are allotted. The intra cluster scheduling algorithm can be written as:

Step 1: Get the Number of Nodes

Step 2: Check for its transmission power

Step 3: If the transmission power is between $15 \mathrm{dbm}$ and $20 \mathrm{dbm}$, the node = IEEE 802.11b (WLAN) node. Assign the operating frequency as $2.413 \mathrm{GHz}$

Step 4: Check for its CBR transmission

Step 5: Assign the transmission time as $5 \mathrm{sec}$ and sleeping time as $100 \mathrm{~m} \mathrm{sec}$

Step 6: If the transmission power is between $0 \mathrm{dbm}$ and $3 \mathrm{dbm}$, the node = IEEE 802.15.4 (WPAN) node. Assign different frequencies for every 5 members cluster. The cluster is with 4 end devices and 1 coordinator
Step 7: Check for its CBR transmission

Step 8: Assign the transmission time as $100 \mathrm{~m} \mathrm{sec}$ and sleeping time as $100 \mathrm{~m} \mathrm{sec}$

Step 9: If the transmission power is not satisfying the above two cases the node is identified as invalid node and assigned sleeping time as total simulation time

The above steps can be written as:

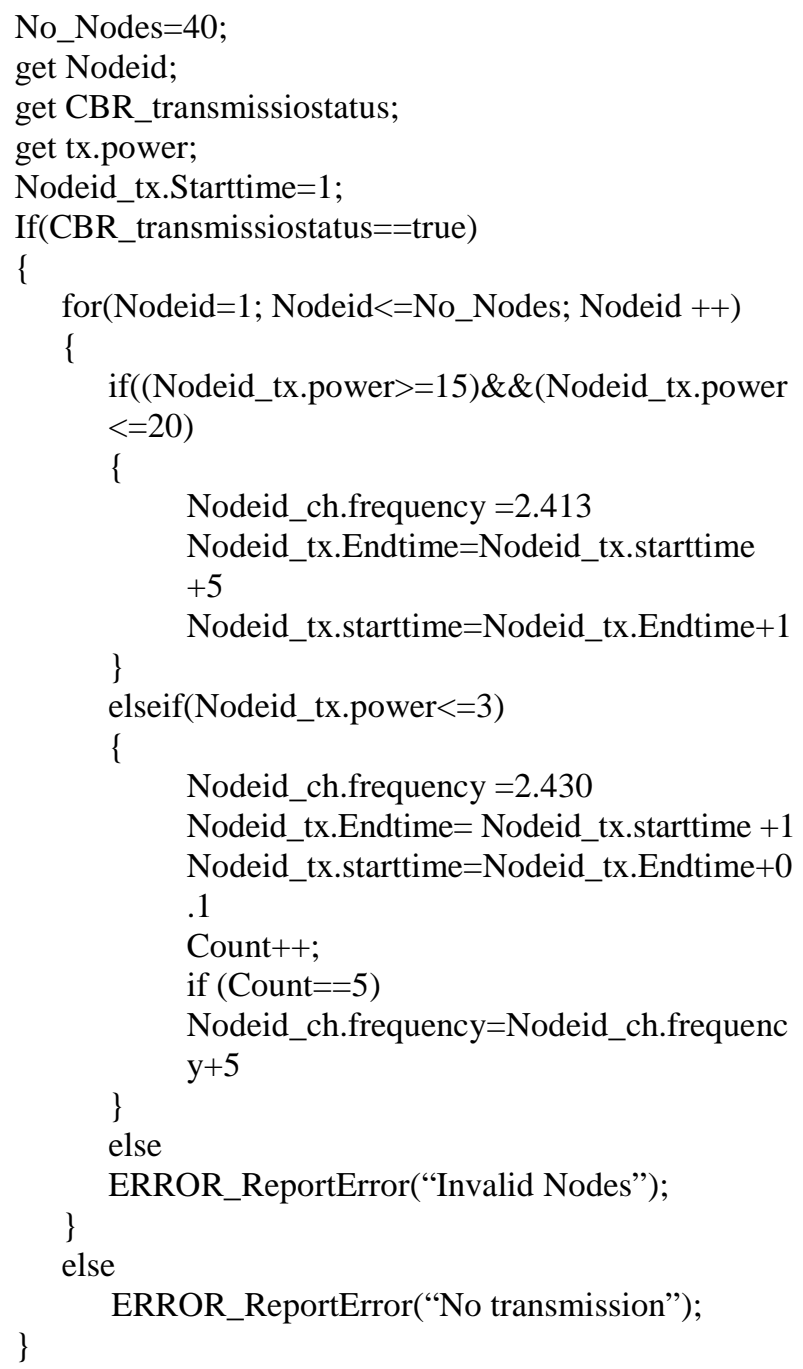

Reformed cluster scheduling: This scheduling scheme is derived from intra cluster scheduling. In reformed cluster scheduling new cluster will be formed from inter cluster transmissions. Transmitting pairs from each cluster will form a new cluster. Instead of having nodes as cluster member, in cluster reformation scheduling the transmission pair from each cluster will form a new cluster. The algorithm is explained as: 
Step 1: Get the Number of Nodes

Step 2: Check for its transmission power

Step 3: If the transmission power is between $15 \mathrm{dbm}$ and $20 \mathrm{dbm}$, the node = IEEE 802.11b (WLAN) node. Assign the operating frequency as $2.413 \mathrm{GHz}$

Step 4: Check for its CBR transmission

Step 5: Assign the transmission time as $5 \mathrm{sec}$ and sleeping time as $100 \mathrm{msec}$

Step 5: If the transmission power is between $0 \mathrm{dbm}$ and $3 \mathrm{dbm}$, the node = IEEE 802.15.4 (WPAN) node. Assign different frequencies for every 5 members cluster. The cluster is with 4 end devices and 1 coordinator

Step 6: Check for its CBR transmission

Step 7: Assign the transmission time as $100 \mathrm{msec}$ and sleeping time as $100 \mathrm{~m} \mathrm{sec}$

Step 8: Check for Error. If error presents get the transmission pair from each cluster and assign frequencies

Step 9: Assign transmission time and sleeping time for new cluster members

Step10: If the transmission power is not satisfying the above two cases the node is identified as invalid node and assigned sleeping time as total simulation time.

The above steps can be written as:

No_Nodes $=40$; get Nodeid;

get CBR_transmissiostatus;

get tx.power;

Nodeid_tx.Starttime $=1$;

If(CBR_transmissiostatus==true)

\{

for(Nodeid=1; Nodeid<=No_Nodes; Nodeid ++)

\{

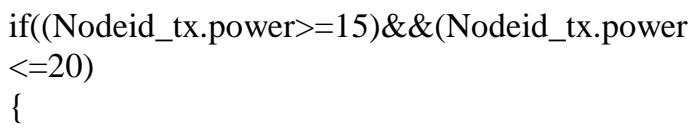

Nodeid_ch.frequency $=2.413$

Nodeid_tx.Endtime $=$ Nodeid_tx.starttime +5 \}

Nodeid_ch.frequency $=2.430$ Nodeid_tx.Endtime $=$ Nodeid_tx.starttime $+1$

Nodeid_tx.starttime=Nodeid_tx.Endtime + 0.1

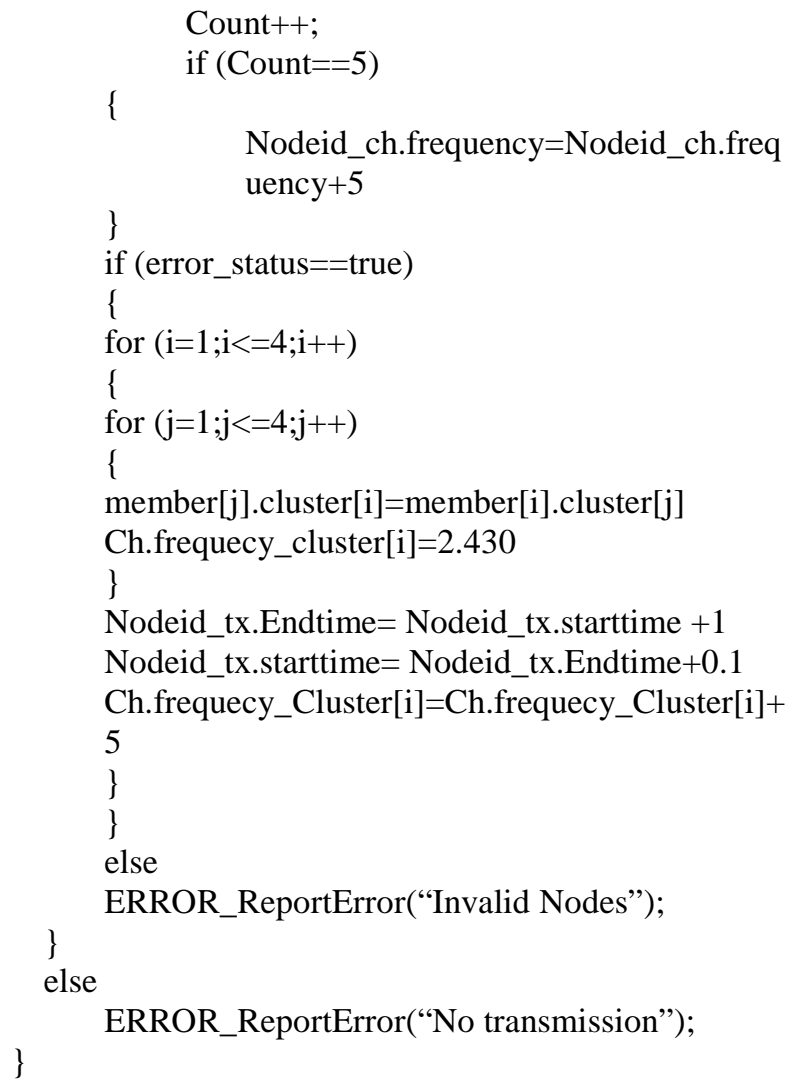

\section{RESULTS}

To evaluate the effectiveness of the proposed scheme in a coexistence heterogeneous wireless network, a simulation study was conducted using Qualnet 4.5 simulator. The simulation is conducted for three different schemes and the results are compared. The bit error is measured from the simulation. The bit error rate is calculated to find Packet Error Rate (PER).For the conventional TDMA scheme all the nodes are linked with single channel and time slots are allotted for transmission. For this scheme the simulation time is fixed as $53 \mathrm{sec}$. The simulation configuration and parameters used in this study is shown in Table 1.

The effectiveness of the proposed scheme was measured with different metrics such as Bit error rate, Packet Error rate, Throughput, Average End-End delay and Average jitter. The Fig. 7-12 shows the performance analysis of random topology with two different proposed schemes.

The Fig. 7 shows the bit error analysis for random topology. In this Fig.7 bit error for conventional TDMA and two different schemes namely intra cluster 


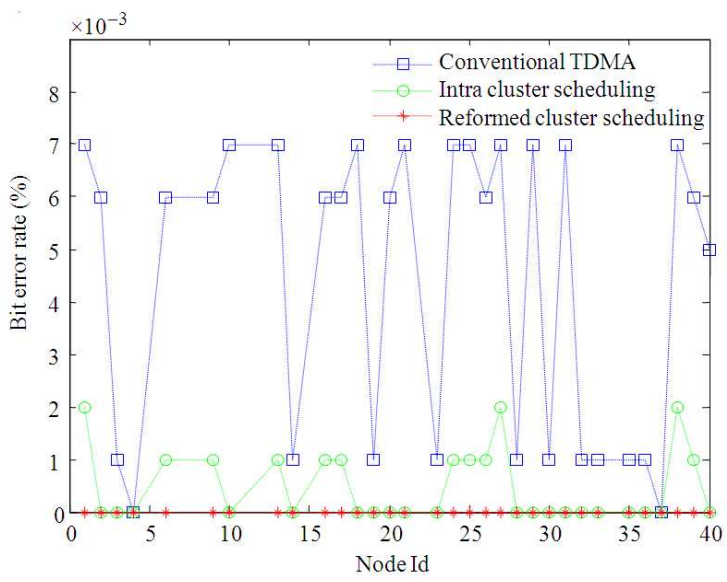

Fig. 7: Bit error rate analysis for random topology

Table 1: Simulation configuration and parameters

\begin{tabular}{lll}
\hline Parameter & IEEE $802.11 \mathrm{~b}$ & IEEE 802.15 .4 \\
\hline Number of nodes & 20 & 20 \\
Transmission power & $15 \mathrm{dbm}$ & $3 \mathrm{dbm}$ \\
Modulation & $\mathrm{CCK}$ & OQPSK \\
MAC protocol & 802.11 & 802.15 .4 \\
Routing protocol & Bellman ford & AODV \\
No of packets & 100 & 100 \\
Payload size & $1500 \mathrm{bytes}$ & $105 \mathrm{bytes}$ \\
Simulation time & $35 \mathrm{sec}$ & \\
Packet interval & $100 \mathrm{~m} \mathrm{sec}$ & $1 \mathrm{~m} \mathrm{sec}$ \\
Packet transmission time & $5 \mathrm{sec}$ & $1 \mathrm{sec}$ \\
Test bed size & $40 \times 40 \mathrm{~m}^{2}$ & \\
Topology & Random & \\
\hline
\end{tabular}

scheduling and reformed cluster scheduling is shown. When the reformed cluster scheduling is adopted the bit error becomes zero. When the conventional TDMA and intra cluster scheduling is adopted time slot mechanism is not helpful in WPAN network because ZigBee is a mesh networking technology, which means that devices can automatically route messages on each other's behalf (often called multi-hopping). This allows deploying larger networks without immoderately increasing the transmission power since direct communications occur only in a geographicallyrestricted area.

The bit error rate analysis for random topology is shown in Fig. 7. When intra cluster scheduling is used the bit error rate is reduced by $71-83 \%$. After implementing reformed cluster scheduling bit error rate becomes zero. Bit error rate is calculated from the successful transmission.

The Fig. 8 shows the packet error rate analysis for random topology. The result shows that the performance of heterogeneous network is improved when the proposed scheme is used. The packet error

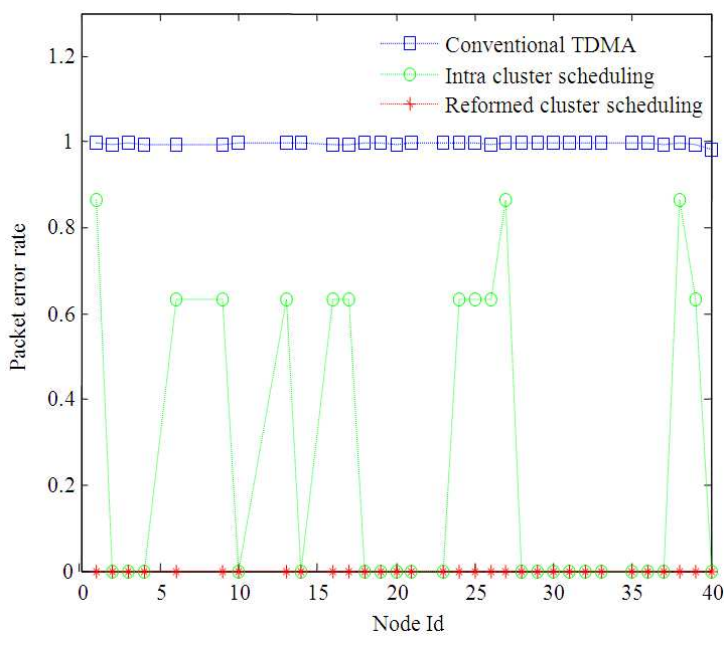

Fig. 8: Packet error rate analysis for random topology

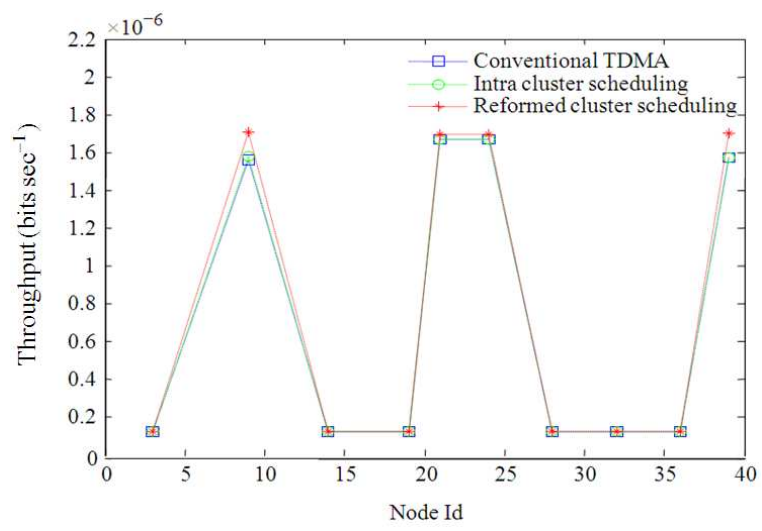

Fig. 9: Throughput analysis for random topology

becomes zero when reformed cluster scheduling is used. The intra cluster scheduling improves the performance by reducing $36 \%$ of the packet error rate.

In Fig. 9 throughput analysis for random topology is shown. The throughput is increased by $1.3 \%$ when intra cluster scheduling is used. After implementing the reformed cluster scheduling, throughput is increased by $9.3 \%$.

In Fig. 8 node id includes both IEEE 802.15.4 and $802.11 \mathrm{~b}$ nodes. Here the random topology is considered. So, the nodes are placed at random and the IEEE 802.15.4 nodes are clustered while the IEEE $802.11 \mathrm{~b}$ nodes are placed around the clusters. In this random topology some of the IEEE $802.11 \mathrm{~b}$ nodes close to the clusters and some of the IEEE $802.11 \mathrm{~b}$ nodes are placed apart. In this part of the research study mobility model is not given much importance because of random topology. 


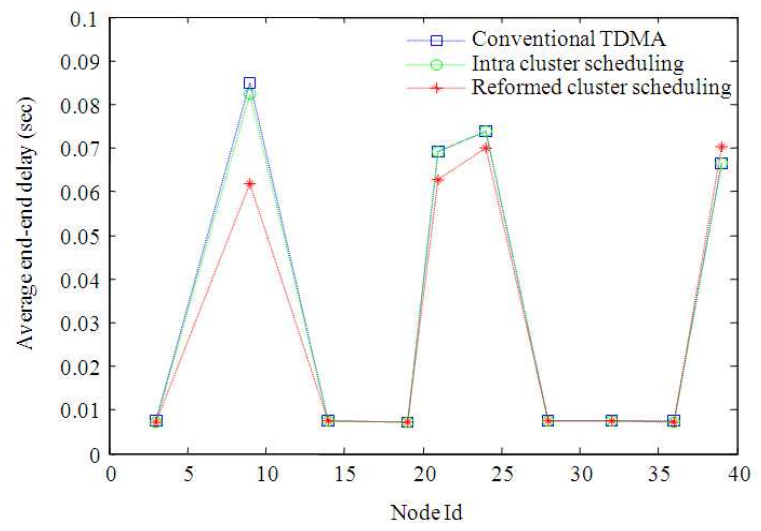

Fig. 10: Average end-end delay analysis for random topology

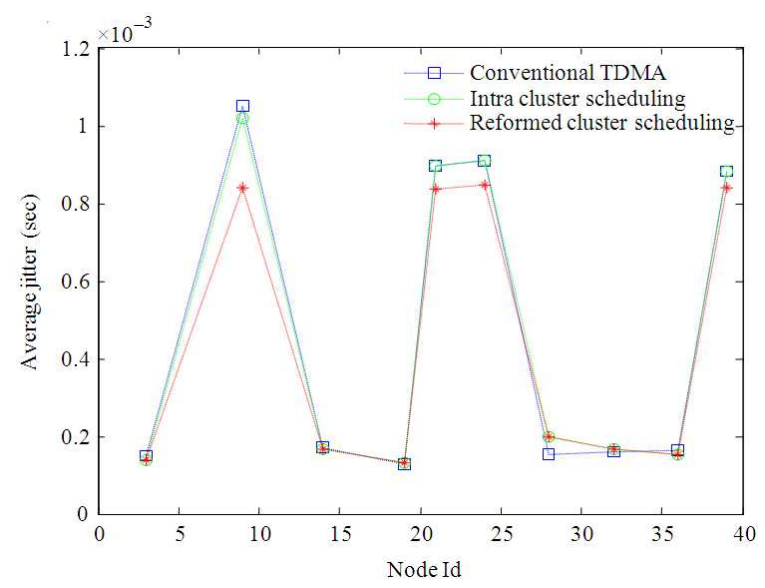

Fig. 11: Average jitter analysis for random topology

The average end-end delay and average jitter analysis for random topology is shown in Fig. 10 and 11 respectively. The end-end delay is decreased by $3 \%$ when intra cluster scheduling is used. When reformed cluster scheduling is used it is decreased by $27 \%$. The average jitter is decreased by $2.8 \%$ when intra cluster scheduling is used. When reformed cluster scheduling is used it is decreased by $20 \%$. Instead of measuring the results for IEEE 802.15.4 nodes the results are plotted for both the IEEE 802.15.4 as well as IEEE $802.11 \mathrm{~b}$ nodes. So that the effect of the proposed scheme for various nodes can be measured easily. In above Fig. 7-11 the comparison of all the three scheduling schemes for various performance measures can be clearly observed for different nodes.

\section{DISCUSSION}

From the three scheduling algorithms the reformed cluster scheduling improves the performance of IEEE 802.15.4. In conventional TDMA scheduling scheme irrespective of the nodes the scheduling is given .In intra cluster scheduling the nodes which are grouped under frequency and transmission power given time slots for packet transmission. In this scheduling scheme the effect of IEEE 802.11b nodes on the performance of IEEE 802.15.4 nodes are negligible but because of mesh networking the time slot mechanism is not helpful for IEEE 802.15.4 nodes. The performance of the intra cluster scheduling algorithm is better than the conventional TDMA scheme because of channel separation. The mesh networking is more complicated, each node may communicate with any other node within range and it requires greater overhead. In reformed cluster scheduling the nodes form each cluster is grouped and allotted unique frequency .The PAN coordinator in the clusters are given frequency switching. In this technique the nodes which are placed within range are separated by frequency. Thereby the interference due to mesh networking is mitigated. This technique is outperforming for indoor environment. From the results the effect of this proposed algorithm in performance improvement of IEEE 802.15.4 is clearly observed.

\section{CONCLUSION}

We in this study present analysis on performance of coexistence heterogeneous networks. In this study, we propose a new power based scheme using intra cluster scheduling and cluster reformation scheduling mechanism for the coexistence of multiple IEEE 802.15.4 LRWPAN and IEEE 802.11b WLAN. The simulation results are compared with the conventional TDMA scheme. The performance metrics of IEEE 802.15.4 network such as bit error rate, packet error rate, throughput, average end-end delay and average jitter is analyzed when the nodes are static. The simulation results show that the proposed scheme is effective in performance improvement for coexistence network of IEEE 802.15.4 for random topology. In future the analysis can be extended with mobility model and the same proposed scheme can be implemented with Exata emulator and free scale processor.

\section{REFERENCES}

Chen, L., T. Sun and M. Gerla, 2006. Modeling channel conflict probabilities between IEEE 802.15 based wireless personal area networks. Proceeding of the IEEE International Conference on Communications, (ICC'06), Academia Sinica, Istanbul, pp: 343-348. DOI: 10.1109/ICC.2006.254751 
Howitt, I. and J. Gutierrez, 2003. IEEE 802.15.4 low rate - wireless personal area network coexistence issues. Proceedings of Wireless Communications and Networking, March 20-20, New Orleans, LA, USA., $\quad$ pp. 1481-1486. DOI: 10.1109/WCNC.2003.1200605

Petrova, M., J. Riihijärvi, P. Mähönen and S. Labella, 2006. Performance study of IEEE 802.15.4 using measurements and simulations. Proceedings of the IEEE Wireless Communications and Networking Conference, (WCNC'06), Las Vegas, Nev, USA., pp: 487-492. DOI: 10.1109/WCNC.2006.1683512

Shin, S.Y., H.S. Parky, S. Choi and W.H. Kwon, 2005a. Lecture notes in computer science: Packet error rate analysis of IEEE 802.15.4 under IEEE 802.11b interference. Lect. Notes Comput. Sci., 3510: 279288. DOI: $10.1007 / 11424505 \_27$
Shin, S.Y., S. Choi, H.S. Park and W.H. Kwon, 2005b. Packet error rate analysis of IEEE 802.15.4 under IEEE $802.11 \mathrm{~b}$ interference. Proceedings of the 3rd International Conference on Wired/Wireless Internet Communications, (WWIC '05), Lecture Notes in Computer Science, Xanthi, Greece, pp: 279-288. DOI: $10.1007 / \mathrm{b} 136247$

Sikora, A. and V.F. Groza, 2005. Coexistence of IEEE 802.15.4 with other Systems in the 2.4GHz-ISMBand. Proceedings of the IEEE Instrumentation and Measurement Technology Conference, May 16-19, University of Cooperative Education, Ottawa, $\quad$ pp: $17-19$ DOI: 10.1109/IMTC.2005.1604479

Yoon, D.G., S.Y. Shin, W.H. Kwon and H.S. Park, 2006. Packet error rate analysis of IEEE $802.11 \mathrm{~b}$ under IEEE 802.15.4 interference. Proceeding of the Vehicular Technology Conference, May 7-10, Seoul Nat. University, Melbourne, pp: 1186-1190. DOI: 10.1109/VETECS.2006.1683022 